Article

\title{
Enumeration of Pentahexagonal Annuli in the Plane
}

\author{
Andrey A. Dobrynin $1,2, *$ (1) and Vladimir R. Rosenfeld ${ }^{3}$ \\ 1 Laboratory of Topology and Dynamics, Novosibirsk State University, Novosibirsk 630090, Russia \\ 2 Laboratory of Graph Theory, Sobolev Institute of Mathematics, Siberian Branch of the Russian Academy of \\ Sciences, Novosibirsk 630090, Russia \\ 3 Department of Computer Science and Mathematics, Ariel University, Ariel 4070000, Israel; \\ vladimir_rosenfeld@yahoo.com \\ * Correspondence: dobr@math.nsc.ru
}

Received: 18 October 2019; Accepted: 22 November 2019; Published: 1 December 2019

\begin{abstract}
Pentahexagonal annuli are closed chains consisting of regular pentagons and hexagons. Such configurations can be easily recognized in various complex designs, in particular, in molecular carbon constructions. Results of computer enumeration of annuli without overlapping on the plane are presented for up to 18 pentagons and hexagons. We determine how many annuli have certain properties for a fixed number of pentagons. In particular, we consider symmetry, pentagon separation (the least ring-distance between pentagons), uniformity of pentagon distribution, and pentagonal thickness (the size of maximal connected part of pentagons) of annuli. Pictures of all annuli with the number of pentagons and hexagons up to 17 are presented (more than 1300 diagrams).
\end{abstract}

Keywords: pentahexagonal annuli; constructive enumeration; nanoarchitecture

\section{Introduction}

Polycyclic molecules are widespread in nature and comprehensively studied in chemistry [1]. Currently, they also cause a growing interest of experts in nanotechnology [2,3]. Among cycles, hexagons are the most frequent elements of molecular constructs. In particular, benzenoid hydrocarbons [4-7], whose carbon skeleton mimics patches of the honeycomb, are entirely composed from fused hexagonal benzene rings. The nearest by size, pentagonal and heptagonal rings (as well as larger ones) are less abundant in nature but they also are incorporated in diverse molecules [8-11]. Since we follow herein a plane graphic model of polycyclic molecules, where all cycles are depicted as regular polygons, it is worth recalling that the plane can unrestrictedly be paved only by congruent regular triangles, squares, and hexagons $[12,13]$. While no other congruent regular polygons of only one type can tile the plane [14]. Here, it is important that there are polycyclic mosaic tilings of plane where cycles may have unequal lengths [12,15]. A surface of a polygonal cage (which consists of polyhedron faces) is effectively a closed mosaic tiling [16,17]. Each plane unfolding of the latter is again similar to a polycyclic molecule. A very important type of polycyclic tilings, by hexagons and pentagons, is due to the discovery of a broad spectrum of fullerene molecules [18]. Herein, we do not restrict our consideration only by patches of fullerene cages $[19,20]$, but we may impose other restrictions on such objects (see later).

Pentahexagonal annuli are molecular graphs of pentahexagonal corannulenes [21-23]. The last chemical term is borrowed from the theory of benzenoid hydrocarbons [4], where it was originally applied to 'simpler molecules' that contain only hexagons. In addition, synonyms of the word 'corannulenes' in organic chemistry are circulenes and primitive (catacondensed) coronoids [24]. Coronoids are regarded by us as substances of potential use to nanotechnology fields (e.g., due to their unique electronic properties; see $[25,26]$ and bibl.). Avoiding the detailed motivation of this statement, we offer here only a few possible examples. In particular, circulenes can be assembled by stacking into columns. 
Owing to the possession of capillary voids, such columns can be used as nanotubules (see $[27,28]$ and bibl.). A continued growth of the columns may produce long hollow fibers. The latter may be (self)woven to produce carpetlike tissues (sheets), which may in turn be rolled up to give (multiwall) nanotubes or the 3-dimensional bulk materials. Thin layered sheets thus obtained may be used as membranes. Such assembled constructs add to ones that are obtained using (strands of) natural DNA/RNA or protein molecules (see [27,29] and bibl.), but have a different chemical nature. A possible area of application (e.g., of the columns) is molecular electronics, where they may, in particular, play a role of semiconductors. And molecular photonics is another such area, where targeted photonic effects are achieved using a respective ordering of molecules (e.g., of corannulenes) in a medium through which passes a beam of light. Such effects (entailed by the controlled ordering/disordering of medium) can be produced using disc-shaped molecules in a (columnar) discotic liquid crystal [30-32], by application of an electric and/or a magnetic field. The simultaneous presence of pentagonal and hexagonal rings, with different proportions thereof, allows one to extend the range of useful properties of envisioned corannulene molecules, due to the difference between the rings.

\section{Classes of Annuli}

A pentahexagonal annulus has a connected part formed from rings that are regular pentagons or hexagons in the plane. Two adjacent rings have an edge in common, and no two nonadjacent rings or three arbitrary ones share a common point. Every ring of an annulus is adjacent with two other rings, i.e., every annulus may be regarded as a closed ribbon consisting of penta- and hexagonal rings (see Figure 1). A pentahexagonal chain is constructed in a similar way, with the only distinction that, therein, there are two end rings having each only one adjacent neighbor. Each annulus (res. chain) made up from $n \geq 2$ rings is obtained by addition of the $n$-th ring to both end rings (res. a fixed end ring) of a certain chain composed of $n-1$ rings.
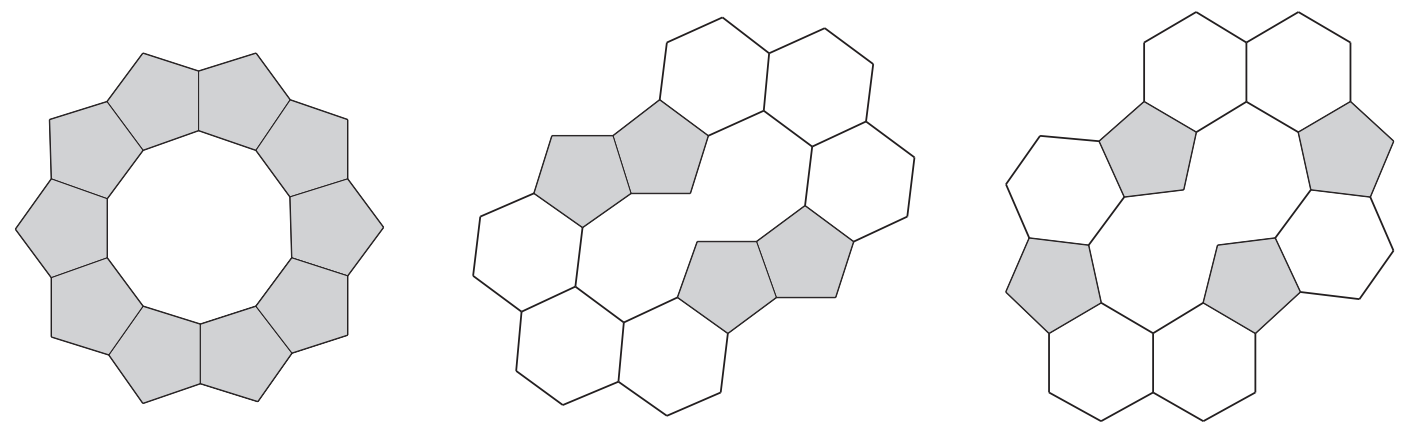

Figure 1. Minimal pentahexagonal annuli, $r=10$.

Denote by $\mathcal{G}_{r}$ the set of all pentahexagonal annuli with $r$ rings without overlapping in the plane. It means that non-adjacent rings have no common points. For the set of all pentahexagonal annuli with $p$ pentagons and $h$ hexagons, we will use notation $\mathcal{G}_{p, h}$,

$$
\mathcal{G}_{r}=\bigcup_{p+h=r} \mathcal{G}_{p, h}
$$

A graph of class $\mathcal{G}_{p, h}$ has $3 p+4 h+2$ vertices and $4 p+5 h+1$ edges. In this paper, we consider pentahexagonal annuli having $p>0$ pentagons.

The generation algorithm of annuli is based on the algorithm of generation of pentahexagonal chains [33]. Every chain with $r$ rings is coded by three binary sequences. The first sequence of length $r$ contains information about sizes of rings ( 5 or 6 ). The other two sequences of length $r-2$ represent two half-boundaries of the chain. They define how the chain grows, namely, the bitwise sum of these sequences determines the acceptable attachment of the rings. During the generation process, coordinates of annulus vertices are calculated. If the terminal rings have two suitable overlapping 
edges, then the edges are fused and an annulus appears. To obtain plane annuli, edge intersections are checked. Since two nonisomorphic chains can give isomorphic annuli, the checking of graph isomorphism is implemented.

Polycyclic structures with rings of various sizes are a subject of constructive or analytical investigation. We mention several studies in this direction. Hexagonal annuli of $\mathcal{G}_{0, r}$ are known under the name 'primitive coronoids'. A comprehensive bibliography on the theory of coronoids can be found in books [24,34]. A mathematical formalization of the theory of coronoids and related structures was developed in [35]. The numbers of helicenic hexagonal chains were reported in [36,37]. Various classes of hexagonal systems were enumerated in [38-43] by constructive and analytical methods. A survey on computer enumeration of polycyclic hexagonal structures was presented in [44]. A fast algorithm for computer generation of polycyclic chains with arbitrary ring sizes was proposed in [45]. Pentahexagonal chains with up to 13 rings were counted with respect to their symmetries in [46], and some problems concerning pentagonal chains and annulus were discussed in [47]. An explicit expression of the counting formula for some hexagonal annuli of the belt-type and Möbius-type was derived in [48]. Certain families of cyclic polyazulenoids consisting of five- and seven-membered rings were enumerated in $[49,50]$.

\section{Structural Properties of Annuli}

The numbers of pentahexagonal annuli of the sets $\mathcal{G}_{r}$ and $\mathcal{G}_{p, h}$ have been obtained for $10 \leq r \leq 18$ where $1 \leq p \leq 18$ and $0 \leq h \leq 17$ by computer calculations. The corresponding data are presented in Table 1. Here, column $r$ contains the numbers of all rings of the considered annuli. The number of all annuli of $\mathcal{G}_{r}$ for the corresponding $r$ is presented in column total. Columns below the letter $p$ contain the cardinality of classes $\mathcal{G}_{p, r-p}$ for the corresponding number of pentagons $p=4,6,8,9, \ldots, 18$ and the number of rings $r$. For example, the number of annuli of class $\mathcal{G}_{6,11}$ with $p=6$ pentagons and $r=17$ rings is equal to 60 .

Table 1. Numbers of pentahexagonal annuli with $r$ rings and $p$ pentagons.

\begin{tabular}{cccccccccccc}
\hline$r$ & \multicolumn{10}{c}{$\boldsymbol{c}$} & Total \\
\cline { 2 - 10 } & $\mathbf{4}$ & $\mathbf{6}$ & $\mathbf{8}$ & $\mathbf{9}$ & $\mathbf{1 0}$ & $\mathbf{1 1}$ & $\mathbf{1 2}$ & $\mathbf{1 4}$ & $\mathbf{1 5}$ & $\mathbf{1 8}$ & \\
\hline 10 & 2 & - & - & - & 1 & & & & & & 3 \\
12 & 11 & 2 & - & - & 1 & - & - & & & & 14 \\
13 & 3 & - & - & - & - & - & - & & & & 3 \\
14 & 61 & 3 & 18 & - & 6 & - & - & 1 & & & 89 \\
15 & 49 & 8 & - & - & 1 & - & - & - & - & & 58 \\
16 & 358 & 32 & 124 & 4 & 33 & - & - & 5 & - & & 556 \\
17 & 515 & 60 & 42 & 1 & 3 & & - & - & - & & 621 \\
18 & 2322 & 309 & 1137 & 1 & 199 & 9 & 104 & 52 & 1 & 5 & 4139 \\
\hline
\end{tabular}

Three minimal graphs form the set $\mathcal{G}_{10}=\mathcal{G}_{10,0} \cup \mathcal{G}_{4,6}$ and their diagrams are depicted in Figure 1 . Diagrams of all graphs of up to 17 pentagons and hexagons are presented as supplement data. The smallest graphs with an odd number of pentagons, $p=9$, appears for 16 rings (see Figure 2). Since fragments of pentagonal chains may have parallel edges, it is easy to construct pentahexagonal annuli with an arbitrary number of rings (see two small pentagonal fragments and an annulus with 22 rings in Figure 3). 

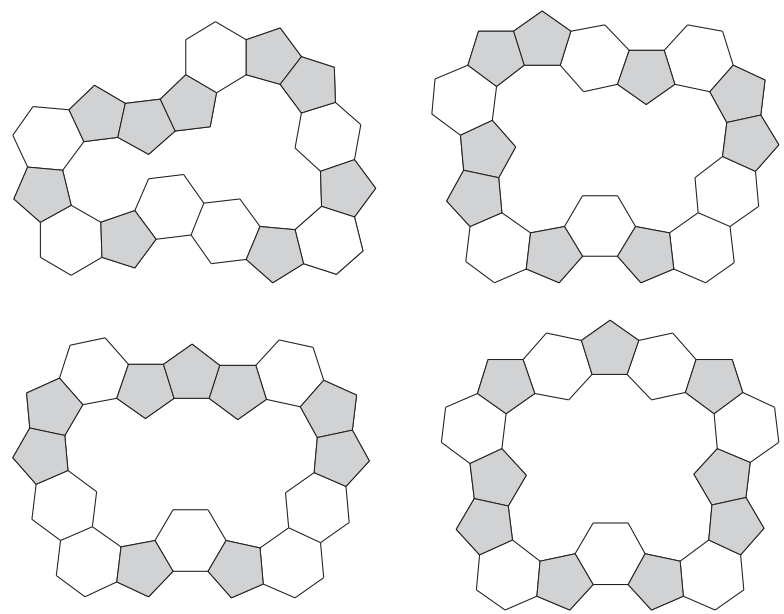

Figure 2. Minimal annuli with an odd number of pentagons, $p=9$.
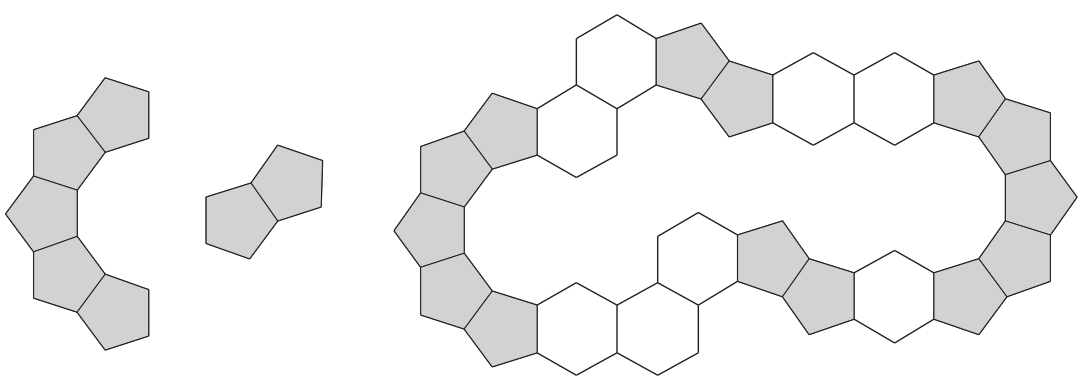

Figure 3. Two pentagonal bricks and a large annulus.

\subsection{Pentagon Separation}

One can see that pentagons are separated by hexagons in various ways. Properties of some complex molecular formations consisting of pentagons and hexagons may depend on location of pentagons relative to each other. For instance, fullerenes where no two pentagons share an edge tend to be more stable [51,52].

By ring-distance between two pentagons of an annulus we mean the minimal number of rings between them. The pentagon separation, $p s(G)$, of an annulus $G$ is the least ring-distance between pentagons of $G$. Pentagon separation of fullerenes is studied in [53]. In particular, if an annulus $G$ has adjacent pentagons, then $p s(G)=0$. In general case, $0 \leq p s(G) \leq\lfloor h / p\rfloor$ for $G \in \mathcal{G}_{p, h}, p \geq 2$. One graph of Figure 1 has $p s(G)=1$, while the others have $p s(G)=0$. We have examined the pentagon separation for the considered families of annuli. Distributions of graphs by their pentagonal separation are given in Table 2. Diagrams of several graphs $G$ with 16 rings and $p s(G)=3$ are shown in Figure 4.

Table 2. Pentagonal separation $p s$ of annuli with $r$ rings.

\begin{tabular}{|c|c|c|c|c|c|}
\hline \multirow{2}{*}{$r$} & \multicolumn{4}{|c|}{$p s$} & \multirow{2}{*}{ Total } \\
\hline & 0 & 1 & 2 & 3 & \\
\hline 10 & 2 & 1 & - & - & 3 \\
\hline 12 & 7 & 5 & 2 & - & 14 \\
\hline 13 & 2 & 1 & - & - & 3 \\
\hline 14 & 55 & 20 & 14 & - & 89 \\
\hline 15 & 36 & 18 & 4 & - & 58 \\
\hline 16 & 348 & 123 & 62 & 23 & 556 \\
\hline 17 & 388 & 172 & 47 & 14 & 621 \\
\hline 18 & 2758 & 846 & 322 & 213 & 4139 \\
\hline
\end{tabular}



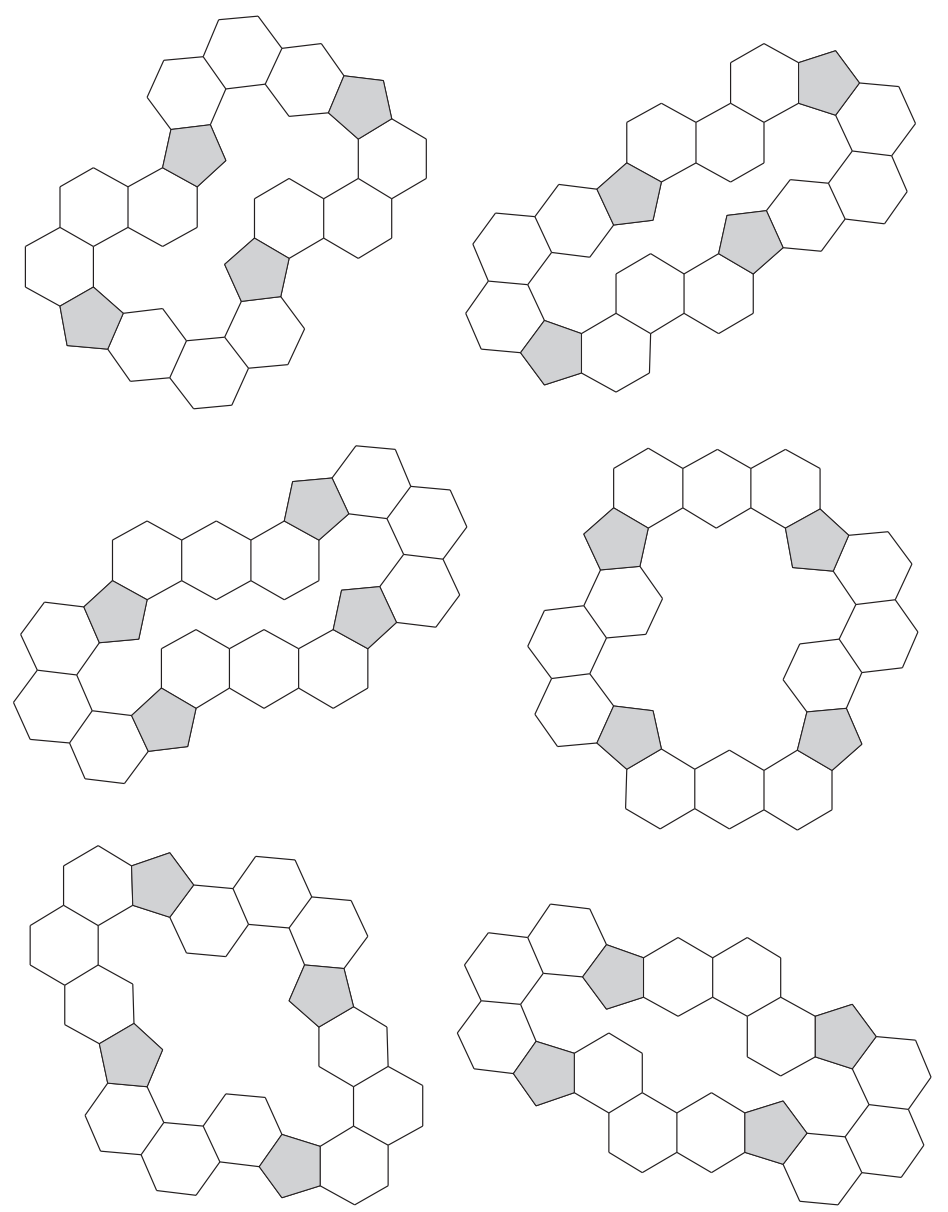

Figure 4. Pentahexagonal annuli of $\mathcal{G}_{4,12}$ with pentagonal separation 3.

\subsection{Pentagon Distribution and Thickness}

A noteworthy structural characteristic of pentahexagonal constructions is the distribution of pentagons between hexagons. This notion comes from studies of fullerenes in which pentagons are distributed as uniformly as possible [54-56]. Sizes of pentagon clusters of fullerenes are studied in [57]. To quantify positions of pentagons in annuli, we introduce a numerical measure based on cyclical structure of annuli. We assume that pentagons are uniformly distributed in an annulus $G$ if its pentagonal separation $p s(G)$ is maximal, i.e., $p s(G)=\lfloor h / p\rfloor, p \geq 2$. Therefore, the length of hexagonal subchains between closest pentagons will be differ in at most 1 ring. A minimal graph with this property has 10 rings (see Figure 1). Table 3 presents the number of graphs of $\mathcal{G}_{p, h}$ with $p$ uniformly distributed pentagons. All graphs of Figure 4 have this property.

Table 3. Annuli with $r$ rings and $p$ uniformly distributed pentagons.

\begin{tabular}{ccccc}
\hline & \multicolumn{3}{c}{$p$} & \\
\cline { 2 - 4 } & $\mathbf{4}$ & $\mathbf{6}$ & $\mathbf{8}$ & \\
\hline 10 & 1 & - & - & 1 \\
12 & 2 & 1 & - & 3 \\
13 & - & - & - & 0 \\
14 & 14 & 1 & - & 15 \\
15 & - & 3 & - & 3 \\
16 & 23 & 4 & 4 & 31 \\
17 & 14 & 6 & - & 20 \\
18 & 183 & 7 & 54 & 244 \\
\hline
\end{tabular}


An opposite and easily recognizable structural characteristic of an annulus is the number of rings of maximal connected part consisting of pentagons only. This quantity will be called the pentagonal thickness, pt $(G)$, of an annulus $G$. Table 4 shows the pentagonal thickness of graphs with $r$ rings. Annuli consisting of pentagons only have maximal pentagonal thickness. An example of graph of $\mathcal{G}_{14,4}$ with pentagonal thickness $p t=6$ is presented in Figure 5. The class of annuli with $p t=1$ contains annuli with uniformly distributed pentagons.

Table 4. Pentagonal thickness $p t$ of annuli with $r$ rings.

\begin{tabular}{|c|c|c|c|c|c|c|c|c|c|c|c|c|c|}
\hline \multirow{2}{*}{$r$} & \multicolumn{12}{|c|}{$p t$} & \multirow{2}{*}{ Total } \\
\hline & 1 & 2 & 3 & 4 & 5 & 6 & 7 & 8 & 9 & 10 & 14 & 18 & \\
\hline 10 & 1 & 1 & - & - & - & - & - & & - & 1 & & & 3 \\
\hline 12 & 7 & 6 & - & - & 1 & - & - & & - & - & & & 14 \\
\hline 13 & 1 & 2 & - & - & - & - & - & & - & - & & & 3 \\
\hline 14 & 34 & 38 & 8 & 6 & 2 & - & - & & - & - & 1 & & 89 \\
\hline 15 & 22 & 36 & - & - & - & - & - & & - & - & - & & 58 \\
\hline 16 & 208 & 248 & 60 & 30 & 5 & - & 4 & & 1 & - & - & & 556 \\
\hline 17 & 233 & 363 & 10 & 13 & 2 & - & - & & - & - & - & & 621 \\
\hline 18 & 1381 & 1930 & 469 & 265 & 48 & 21 & 15 & 2 & 3 & - & - & 5 & 4139 \\
\hline
\end{tabular}

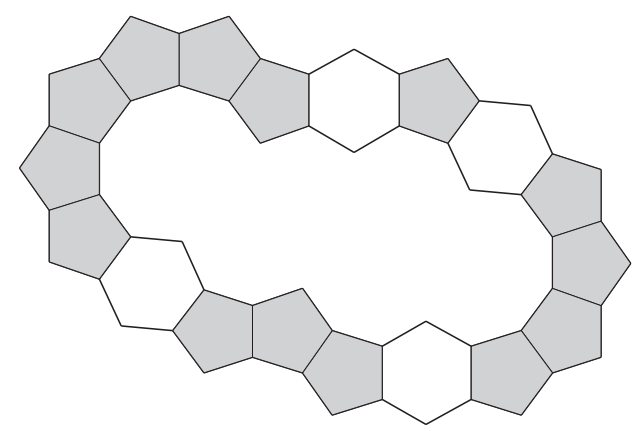

Figure 5. Annuli with 18 rings and pentagonal thickness 6 .

\subsection{Symmetry of Annuli}

Molecular symmetry is a fundamental concept in chemistry, as it can predict or explain many of a molecule's chemical properties. A molecular graph has symmetry if it has a non-trivial automorphism. Some problems related to symmetry of benzenoid systems and fullerenes are discussed in [58-62]. Synthesis and aromaticity of certain annuli with high symmetry are described in $[25,26]$. We have determined how many annuli possess symmetry (without detailed classifications). The numbers of graphs of $\mathcal{G}_{r}$ having symmetries are presented in Table 5. For example, all minimal graphs of $\mathcal{G}_{10}$ have non-trivial symmetries (see Figure 1). Several graphs of $\mathcal{G}_{18}$ with high symmetry are depicted in Figure 6 . The symmetry groups of these annuli are indicated near the diagrams.

Table 5. Numbers of symmetrical annuli with $p$ pentagons.

\begin{tabular}{cccccccccccc}
\hline & \multicolumn{10}{c}{$p$} & Total \\
\cline { 2 - 9 } & $\mathbf{4}$ & $\mathbf{6}$ & $\mathbf{8}$ & $\mathbf{9}$ & $\mathbf{1 0}$ & $\mathbf{1 1}$ & $\mathbf{1 2}$ & $\mathbf{1 4}$ & $\mathbf{1 5}$ & $\mathbf{1 8}$ & \\
\hline 10 & 2 & - & - & - & 1 & & & & & & 3 \\
12 & 10 & 2 & - & - & 1 & - & - & & & & 13 \\
13 & - & - & - & - & - & - & - & & & & 0 \\
14 & 39 & - & 18 & - & 6 & - & - & 1 & & & 64 \\
15 & - & 6 & - & - & 1 & - & - & - & - & & 7 \\
16 & 142 & 3 & 97 & 2 & 26 & - & - & 4 & - & & 274 \\
17 & - & - & 10 & - & 3 & - & - & - & - & & 13 \\
18 & 499 & 26 & 574 & - & 118 & 1 & 93 & 40 & 1 & 5 & 1357 \\
\hline
\end{tabular}



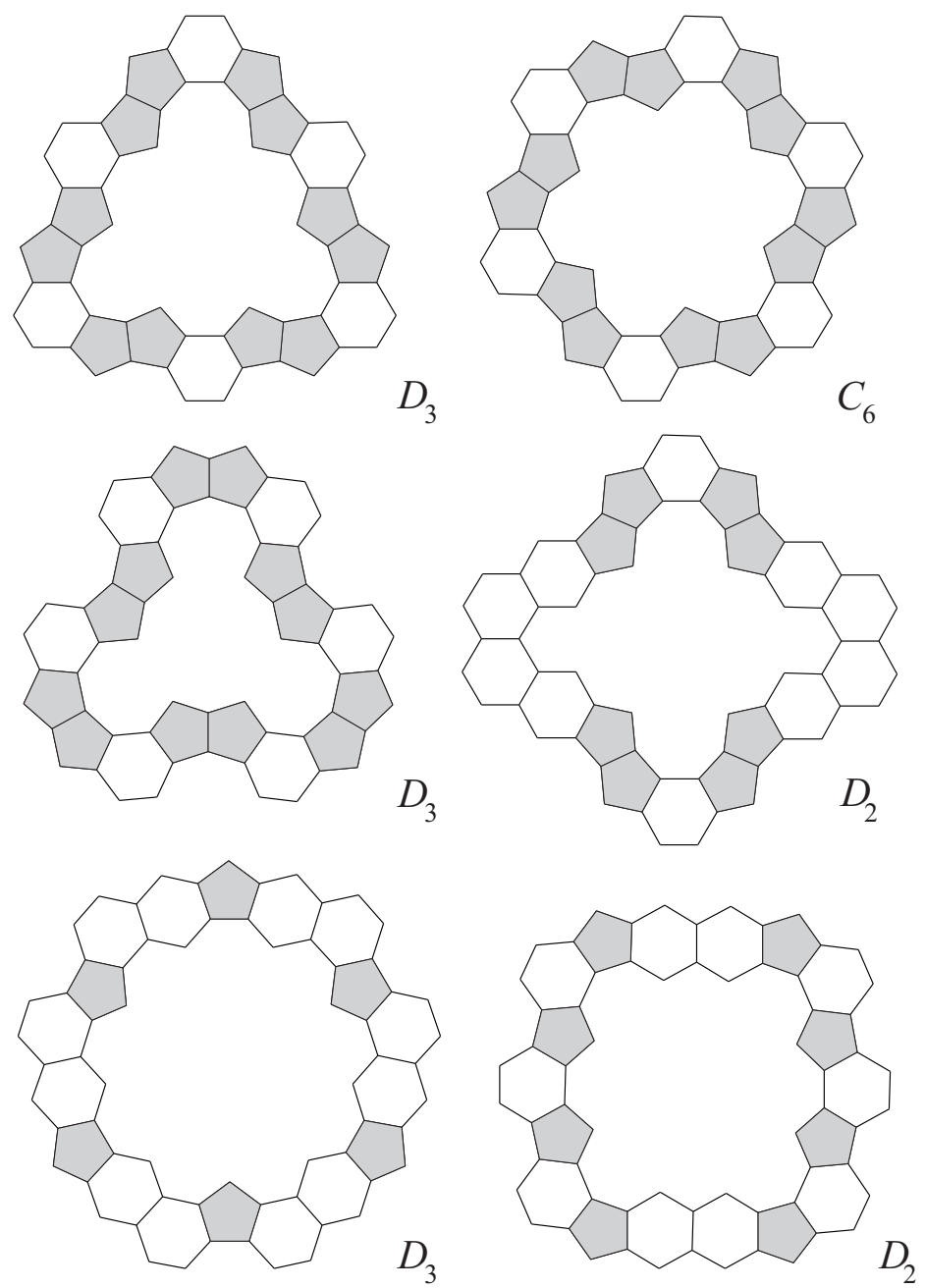

Figure 6. Some symmetric pentahexagonal annuli with 18 rings.

\section{Complex Constructions}

Pentahexagonal annuli can be used for building complex constructions in the plane. It may concern a molecular self-assembly process in which components, either separate or linked, spontaneously form ordered aggregates [63]. Here, we present some examples of infinitely growing configurations. The both annuli of $\mathcal{G}_{4,6}$ are suitable for plane covering as illustrated in Figure 7 . In these cases, annuli connect with each other with overlapping rings. The configuration of Figure 7a is formed from identically oriented annuli, while the configuration of Figure $7 \mathrm{~b}$ contains annuli connected in two ways. Two configurations with rotational symmetry are shown in Figures 8 and 9. These graphs can be built by fusing edges of hexagonal rings of annuli. 


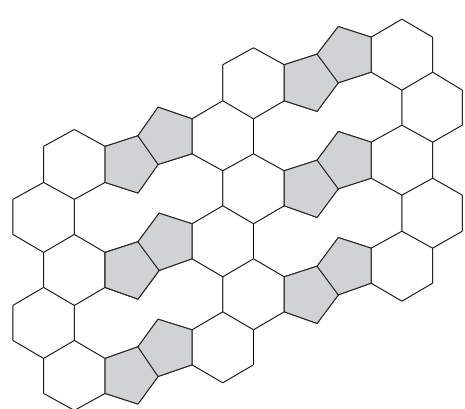

(a)

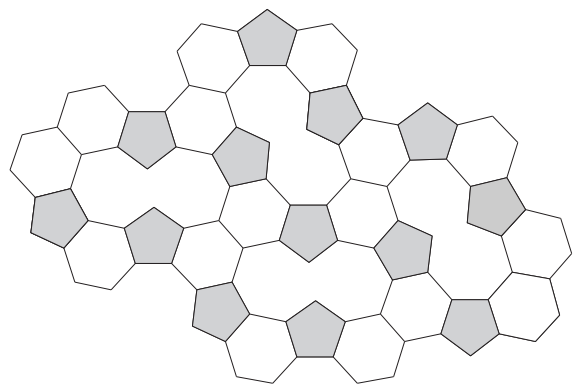

(b)

Figure 7. Two configurations generated by annuli with overlapping rings. (a) Identically oriented annuli; (b) Annuli connected in two ways.

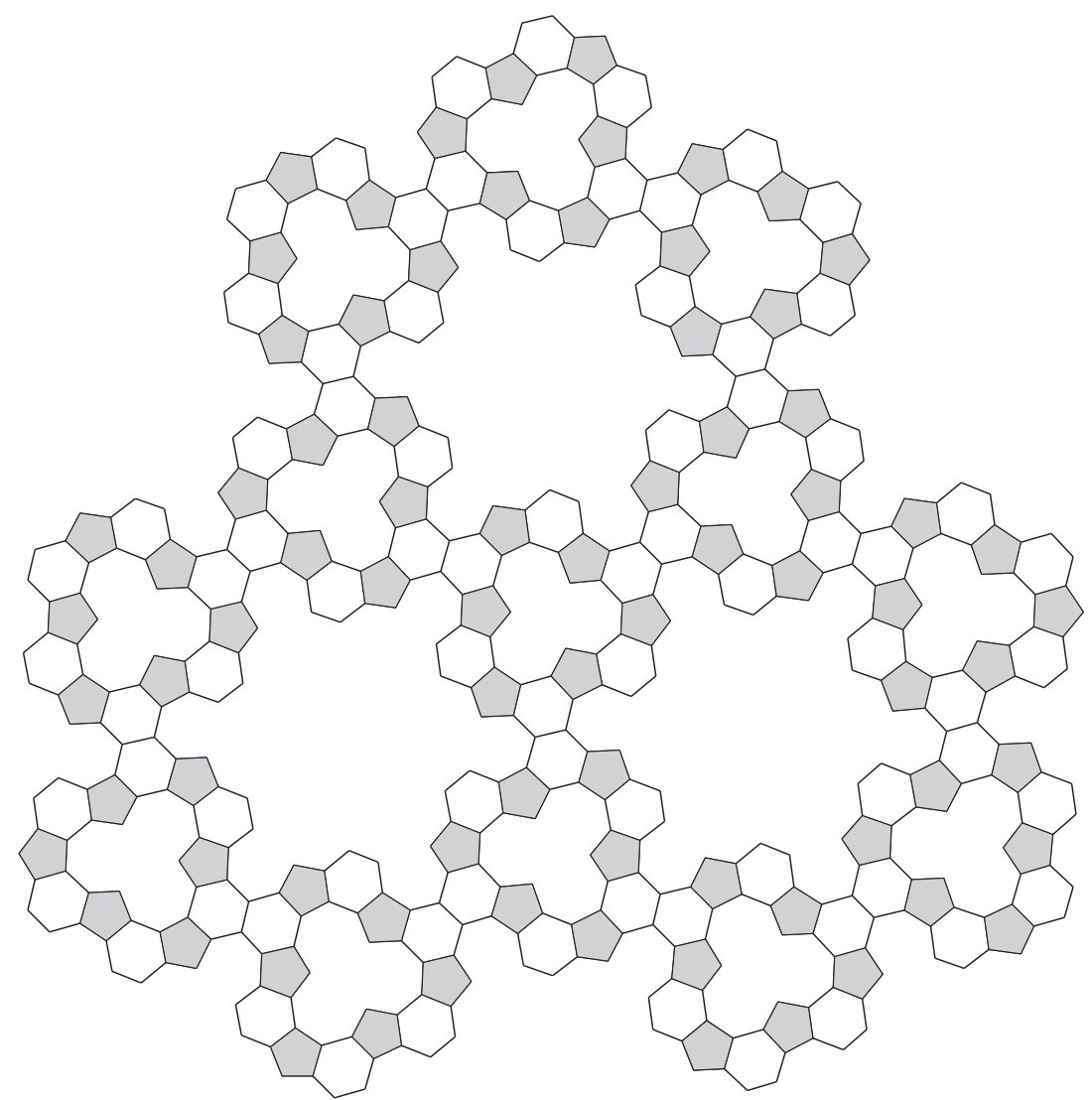

Figure 8. 13 annuli of $\mathcal{G}_{6,6}$ fused by edges. 


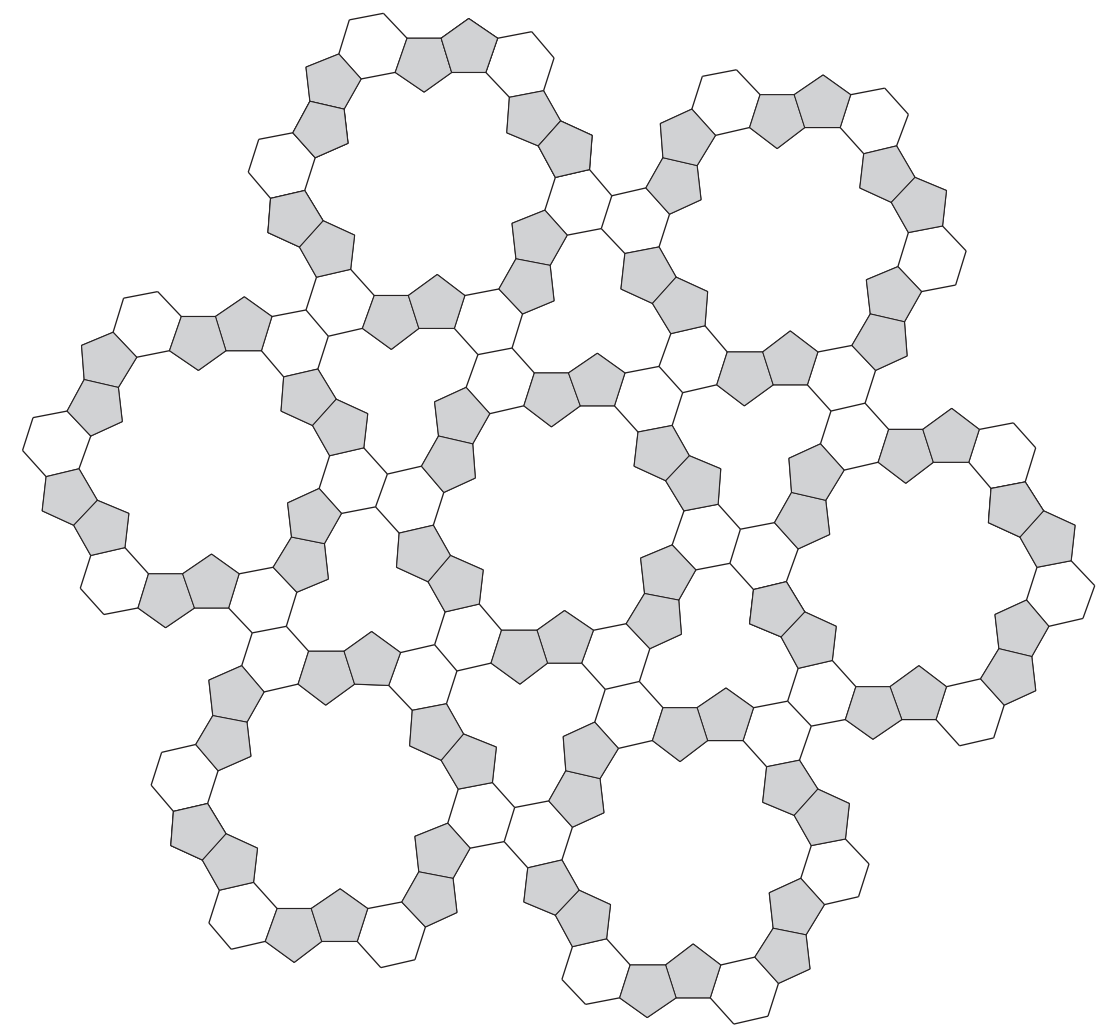

Figure 9. 7 annuli of $\mathcal{G}_{12,6}$ fused by edges.

\section{Conclusions}

Pentahexagonal annuli can be considered as parts of various molecular complex carbon structures. In this study, we have enumerated annuli without overlapping in the plane of up to 18 pentagons and hexagons. We have counted how many annuli with a fixed number of pentagons have certain properties, such as symmetry, pentagon separation, pentagonal thickness, and uniform distribution of pentagons. The obtained numerical results are collected in Tables 1-5. The diagrams of annuli were depicted in decreasing order of the number of pentagons up to 17 rings. It is shown that some complex planar assemblies can be formed from pentahexagonal annuli.

Author Contributions: Methodology, V.R.R.; Software, A.A.D.

Funding: This work was supported by the Laboratory of Topology and Dynamics, Novosibirsk State University (contract no. 14.Y26.31.0025 with the Ministry of Education and Science of the Russian Federation).

Conflicts of Interest: The authors declare no conflict of interest.

\section{References}

1. Orchin, M.; Macomber, R.S.; Pinhas, A.R.; Wilson, R.M. The Vocabulary and Concepts of Organic Chemistry, 2nd ed.; John Wiley \& Sons, Inc.: Hoboken, NJ, USA, 2005.

2. Allis, D.G.; Spencer, J.T. Handbook of Nanoscience, Engineering, and Technology; Chapter Nanostructural Architectures from Molecular Building Blocks; CRC Press: Boca Raton, FL, USA, 2003; pp. 1601-1667.

3. Lehn, J.M. Supamolecular Chemistry —Concepts and Perspectives; VCH Verlagsgesellschaft: Weinheim, NY, USA, 1995.

4. Balaban, A.T. Challenging problems involving benzenoid polycycles and related systems. Pure Appl. Chem. 1982, 54, 1075-1096. [CrossRef]

5. Gutman, I.; Cyvin, S.J. Introduction to the Theory of Benzenoid Hydrocarbons; Springer: Berlin, Germany, 1989.

6. Gutman, I.; Cyvin, S.J. (Eds.) Advances in the Theory of Benzenoid Hydrocarbons; Springer: Berlin, Germany, 1990; Volume 153. 
7. Gutman, I. (Ed.) Advances in the Theory of Benzenoid Hydrocarbons II; Springer: Berlin, Germany, 1992; Volume 162.

8. Deza, M.; Fowler, P.W.; Shtogrin, M.; Vietze, K. Pentaheptite modifications of the graphite sheet. J. Chem. Inf. Comput. Sci. 2000, 40, 1325-1332. [CrossRef] [PubMed]

9. Pop, R.; Medeleanu, M.; Diudea, M.V.; Szefler, B.; Cioslowski, J. Fullerenes patched by flowers with octagonal core. Cent. Eur. J. Chem. 2014, 12, 90-97. [CrossRef]

10. Sundholm, D.; Wirz, L.N.; Schwerdtfeger, P. Novel hollow all-carbon structures. Nanoscale 2015, 7, 15886-15894. [CrossRef] [PubMed]

11. Zhang, S.; Zhou, J.; Wang, Q.; Chen, X.; Kawazoe, Y.; Jena, P. Penta-graphene: A new carbon allotrope. Proc. Natl. Acad. Sci. USA 2015, 112, 2372-2377. [CrossRef]

12. Grünbaum, B.; Shephard, G.C. Tilings by regular polygons. Patterns in the plane from Kepler to the present, including recent results and unsolved problems. Math. Mag. 1977, 50, 227-247. [CrossRef]

13. Kuperberg, D.; Kuperberg, W. Double-lattice packings of convex bodies in the plane. Discret. Comput. Geom. 1990, 5, 389-397. [CrossRef]

14. Bagina, O. Tiling the plane with congruent equilateral convex pentagons. J. Comb. Theory Ser. A 2004, 105, 221-232. [CrossRef]

15. Zhu, H.; Balaban, A.T.; Klein, D.J.; Živković, T.P. Conjugated-circuit computations on two-dimensional carbon networks. J. Chem. Phys. 1994, 101, 5281-5292. [CrossRef]

16. Fetter, H.L.; Arredondo Ruíz, J.H. On the overlappings in the unfoldings of the dodecahedron. J. Math. Sci. Adv. Appl. 2011, 9, 1-20.

17. Pandey, S.; Ewing, M.; Kunas, A.; Nguyen, N.; Gracias, D.H.; Menon, G. Algorithmic design of self-folding polyhedra. Proc. Natl. Acad. Sci. USA 2011, 108, 19885-19890. [CrossRef] [PubMed]

18. Fowler, P.W.; Manolopoulos, D.E. An Atlas of Fullerenes; Dover Publications Inc.: New York, NY, USA, 2006.

19. Lord, E.A.; Mackay, A.L.; Ranganathan, S. New Geometries for New Materials; Cambridge University Press: Cambridge, UK, 2006.

20. Sadoc, J.F.; Mosseri, R. Geometrical Frustration; Cambridge University Press: Cambridge, UK, 1999.

21. Jenny, W.; Peter, R. Coronaphene, eine neue Gruppe cyclischer Kohlenwasserstoffe. Angew. Chem. 1965, 77, 1027. [CrossRef]

22. Barth, W.E.; Lawton, R.G. Dibenzo[ghi,mno]fluoranthene. J. Am. Chem. Soc. 1966, 88, 380-381. [CrossRef]

23. Barth, W.E.; Lawton, R.G. The synthesis of corannulene. J. Am. Chem. Soc. 1971, 93, 1730-1745.

24. Cyvin, S.J.; Brunvoll, J.; Chen, R.S.; Cyvin, B.N.; Zhang, F.J. Theory of Coronoid Hydrocarbons II; Number 62 in Lecture Notes in Chem.; Springer: Berlin, Germany, 1994.

25. Liu, C.; Sandoval-Salinas, M.E.; Hong, Y.; Gopalakrishna, T.Y.; Phan, H.; Aratani, N.; Herng, T.S.; Ding, J.; Yamada, H.; Kim, D.; et al. Macrocyclic polyradicaloids with unusual super-ring structure and global aromaticity. Chem 2018, 4, 1586-1595. [CrossRef]

26. Stępień, M. An aromatic riddle: Decoupling annulene conjugation in coronoid cacrocycles. Proc. Natl. Acad. Sci. USA 2018, 4, 1481-1483. [CrossRef]

27. Heddle, J.G. Protein cages, rings and tubes: useful components of future nanodevices? Nanotechnol. Sci. Appl. 2008, 1, 67-78. [CrossRef]

28. Rosenfeld, V.R. The fractal nature of folds and the Walsh copolymers. J. Math. Chem. 2016, 54, 559-571. [CrossRef]

29. Ke, Y.; Douglas, S.M.; Liu, M.; Sharma, J.; Cheng, A.; Leung, A.; Liu, Y.; Shih, W.M.; Yan, H. Multilayer DNA origami packed on a square lattice. J. Am. Chem. Soc. 2009, 131, 15903-15908. [CrossRef]

30. Chandrasekhar, S.; Sadashiva, B.K.; Suresh, K.A. Liquid crystals of disc-like molecules. Pramana 1977, 9, 471-480. [CrossRef]

31. Laschat, S.; Baro, A.; Steinke, N.; Giesselmann, F.; Hagele, C.; Scalia, G.; Judele, R.; Kapatsina, E.; Sauer, S.; Schreivogel, A.; et al. Discotic liquid crystals: from tailor-made synthesis to plastic electronics. Angew. Chem. Int. Ed. 2007, 46, 4832-4887. [CrossRef] [PubMed]

32. Lin, L. Bowlic liquid crytals. Mol. Cryst. Liq. Cryst. 1987, 146, 41-54.

33. Dobrynin, A.A. Generation of graphs of unbranched pentagonal and pentahexagonal systems. Vychisl. Sist. 1991, 140, 143-206. (In Russian)

34. Cyvin, S.J.; Brunvoll, J.; Cyvin, B.N. Theory of Coronoid Hydrocarbons; Number 54 in Lecture Notes in Chem.; Springer: Berlin, Germany, 1991. 
35. Bašić, N.; Fowler, P.W.; Pisanski, T. Coronoids, patches and generalized altans. J. Math. Chem. 2016, 54, 977-1009. [CrossRef]

36. Dobrynin, A.A. Enumeration of some subclasses of graphs of unbranched hexagonal systems. Vychisl. Sist. 1990, 136, 16-34. (In Russian)

37. Zhang, F.; Brunvoll, J.; Cyvin, S.J.; Cyvin, B.N.; Guo, X.; Dobrynin, A.A. Theory of helicenic hydrocarbons. Part 5: irreducible helicenes and their enumeration. Struct. Chem. 1996, 7, 263-271.

38. Balaban, A.T.; Harary, F. Chemical graphs-V: Enumeration and proposed nomenclature of benzenoid cata-condensed polycyclic aromatic hydrocarbons. Tetrahedron 1968, 24, 2505-2516. [CrossRef]

39. He, W.; He, W. Generation and enumeration of planar polycyclic aromatic hydrocarbons. Tetrahedron 1986, $42,5291-5299$.

40. Knop, J.V.; Müller, W.R.; Szymanski, K.; Trinajstić, N. Computer Generation of Certain Classes of Molecules; SKTH/Kemija u Industriji: Zagreb, Croatia, 1985.

41. Ratko, T.; Kovačevič, M. Generating and counting unbranched catacondensed benzenoids. J. Chem. Inf. Comput. Sci. 1988, 1, 29-31.

42. Trinajstić, N.; Nikolić, S.; Knop, J.V.; Müller, W.R.; Szymanski, K. Computational Chemical Graph Theory: Characterization, Enumeration, and Generation of Chemical Structures by Computer Methods; Ellis Horwood: New York, NY, USA, 1991.

43. Vöge, M.; Guttmann, A.J.; Jensen, I. On the number of benzenoid hydrocarbons. J. Chem. Inf. Comput. Sci. 2002, 42, 456-466. [CrossRef]

44. Brinkmann, G.; Caporossi, G.; Hansen, P. A survey and new results on computer enumeration of polyhex and fusene hydrocarbons. J. Chem. Inf. Comput. Sci. 2003, 43, 842-851. [CrossRef] [PubMed]

45. Brinkmann, G.; Dobrynin, A.A.; Krause, A. Fast generation of polycyclic chains with arbitrary ring sizes. MATCH Commun. Math. Comput. Chem. 2000, 41, 137-144.

46. Dobrynin, A.A. Generation of graphs of unbranched pentahexagonal catacondensed systems. Croat. Chem. Acta 1993, 66, 91-100.

47. Rosenfeld, V.R.; Dobrynin, A.A.; Oliva, J.M.; Rue, J. Pentagonal chains and annuli as models for designing nanostructures from cages. J. Math. Chem. 2016, 54, 765-776. [CrossRef]

48. Qian, J.; Zhang, F. Counting the cyclocized polyphenacenes. J. Comput. Chem. 2010, 31, 2577-2584. [CrossRef] [PubMed]

49. Deng, K.; Zhang, X. Enumeration of cyclic polyazulenoids. J. Math. Chem. 2016, 54, 416-427. [CrossRef]

50. Zhang, X.; Deng, K. Enumeration of Möbius type cyclic polyazulenoids. J. Math. Chem. 2017, 55, $132-141$. [CrossRef]

51. Albertazzi, E.; Domene, C.; Fowler, P.W.; Theine, T.; Sievert, G.; Van Alieno, C.; Terzetto, F. Pentagon adjacency as a determinant of fullerene stability. Phys. Chem. Chem. Phys. 1999, 1, 2913-2918. [CrossRef]

52. Schmalz, T.G.; Seitz, W.A.; Klein, D.J.; Hite, G.E. Elemental carbon cages. J. Am. Chem. Soc. 1988, 110, 1113-1127. [CrossRef]

53. Goedgebeur, J.; McKay, B.D. Fullerenes with distant pentagons. MATCH Commun. Math. Comput. Chem. 2015, 74, 659-672.

54. Raghavachari, K. Ground state of C84: Two almost isoenergetic isomers. Chem. Phys. Lett. 1992, 190, $397-400$. [CrossRef]

55. Rodriguez-Fortea, A.; Alegret, N.; Balch, A.L.; Poblet, J.M. The maximum pentagon separation rule provides a guideline for the structures of endohedral metallofullerenes. Nat. Chem. 2010, 2, 955-961. [CrossRef] [PubMed]

56. Schein, S.; Friedrich, T. A geometric constraint, the head-to-tail exclusion rule, may be the basis for the isolated-pentagon rule in fullerenes with more than 60 vertices. Proc. Natl. Acad. Sci. USA 2008, 105, 19142-19147. [CrossRef] [PubMed]

57. Bašić, N.; Brinkmann, G.; Fowler, P.W.; Pisanski, T.; Van Cleemput, N. Sizes of pentagonal clusters in fullerenes. J. Math. Chem. 2017, 55, 1669-1682. [CrossRef]

58. Babić, D.; Klein, D.J.; Sah, C.H. Symmetry of fullerenes. Chem. Phys. Lett. 1993, 211, 235-241. [CrossRef]

59. Knor, M.; Škrekovski, R.; Tepeh, A. Mathematical aspects of Wiener index. ARS Math. Contemp. 2016, 11, 327-352. [CrossRef]

60. Kovič, J.; Pisanski, T.; Balaban, A.T.; Fowler, P.W. On symmetries of benzenoid systems. MATCH Commun. Math. Comput. Chem. 2014, 72, 3-26. 
61. Mandal, B.; Banerjee, M.; Mukherjee, A.K. Construction of planar graphs for IPR fullerenes using 5- and 6-fold rotational symmetry: Some eigenspectral analysis. Phys. Chem. Chem. Phys. 2004, 6, 2040-2043. [CrossRef]

62. Schwerdtfeger, P.; Wirz, L.N.; Avery, J. The topology of fullerenes. WIREs Comput. Mol. Sci. 2015, 5, 96-145. [CrossRef]

63. Whitesides, G.M.; Boncheva, M. Beyond molecules: self-assembly of mesoscopic and macroscopic components. Proc. Natl. Acad. Sci. USA 2002, 99, 4769-4774. [CrossRef]

(C) 2019 by the authors. Licensee MDPI, Basel, Switzerland. This article is an open access article distributed under the terms and conditions of the Creative Commons Attribution (CC BY) license (http://creativecommons.org/licenses/by/4.0/). 